\begin{tabular}{|c|ccc|c|c|}
\hline \multicolumn{3}{|c|}{ PORT SAID ENGINEERING RESEARCH JOURNAL } \\
\hline \\
\hline
\end{tabular}

\title{
The Optimal Classification of Partial Discharge Defects within XLPE Cable by Using ANN and Statistical Techniques
}

\author{
Sobhy S. Dessouky ${ }^{1}$, Adel A. El Faraskoury ${ }^{2}$, S. M. El-Mekkawy ${ }^{3}$, and Waleed. Elzanati ${ }^{4}$
}

\begin{abstract}
The classification of medium voltage cable defects is the most important tool to avoid the inaccurate Partial Discharge (PD) measurements. This paper presents a proposed methodology based on pattern recognition technique to classify the PD occurred in cables into two distinct groups: internal and external. The Artificial Neural Networks (ANNs) with different input schemes have been built to obtain the optimal classification. Many statistical features, which extracted by different techniques from measurements, have established the input schemes 3D-pattern PD to improve the performance and classification speed of ANN. In order to obtain the effective statistical features, the study and comparison between all ANN has been finished by evaluating the classification through two parameters: the mean square error (MSE) and the accuracy of neural network. As a result, the proposed approach provides high recognition rate of classification between internal and external PD within Cross-Linked Polyethylene (XLPE) insulated medium voltage cable.
\end{abstract}

Keywords: Partial Discharge, XLPE Power Cables, Pattern Classification, Neural Network, Statistical features

\section{Introduction}

The classification of PD is a necessity to identify the discharges of unknown origin and those caused by defects in the XLPE insulation of medium voltage power cable. The classification of the defects can also be considered as a diagnostic tool. The classification of defects can provide vital clues on the health of the dielectric. In addition, the fast classification into internal and external PD is used in the laboratory tests result to avoid the inaccurate PD measurements. Where, the decision of repairing cable terminations for cable under PD measurements will be immediately undertaken in the situation of observed external PD.

Based on the place of occurrence, in power cable, partial discharge can be classified into two types: internal discharges external (corona) discharges. Internal discharge occurs in gas-filled cavities inside cable insulation near contaminants. External (corona) discharge occurs around sharp conducting points between metal and air. Corona discharge detected from the cable are produced outside the cable and causes inaccurate diagnosis. Every defect that causes PD may have a direct impact on the three dimensional (3D) PD patterns. Therefore, a method aided by a computer program for classifying various types of PD can reduce the labour and expertise required for making such classifications.

\footnotetext{
TSobhy S. Dessouky is with Department of Electrical Engineering Port Said University, Port Said, Egypt

(e-mail: sobhyserry@yahoo.com).

${ }^{2}$ Adel A. El Faraskoury is with Egyptian Electricity Holding Company,

Extra High Voltage Research Centre, Cairo, Egypt

(e-mail: dr.adel_elfaraskoury@yahoo.com).

${ }^{3}$ S. M. El-Mekkawy is with Department of Electrical Engineering Port Said University, Port Said, Egypt (e-mail: makkawy06@hotmail.com).

${ }^{4}$ Waleed M. Elzanati is with Department of Electrical Engineering -

Port Said University, Port Said, Egypt

(e-mail: waleed_elzanaty@yahoo.com).
}

The method combined with a computer program will use extraction techniques to retrieve features from the PD patterns to meet the input requirements for a neural network.In the past, classification of discharge patterns was performed visually by monitoring the patterns on an oscilloscope. Monitoring was done without the involvement of mathematic aspect, with low accuracy. Therefore, nowadays, the use of statistical parameters in the classification work has become the preferred classification technique to replace the old style of classification [1]. Sources of PD are the systems and/or apparatuses operate at high voltage. The defect of PD has been observed in XLPE cables and a crash can cause an interruption in electricity supply and result in a loss of considerable profits.

The measurements of PD are classified by many various techniques including fuzzy clustering [2] and the most popular probabilistic neural network PNN technique [3-4]. The ANN technique is a quick and more stable for the classification of learning styles, and input adjustment operations allow access to important new information [5]. Therefore, the ANN techniques and the statistical parameters are efficiently used in researches [6-10]. The authors have developed a new technique by extracting statistical features from PD experimentally results.

This paper presents a pattern classification technique based on the classification of the internal and the external PD through three layers feed-forward ANNXLPE medium voltage cable. The input data for the classification system is the measurements of three dimensional ( $\varphi-q-n)$ patterns of cable defects and database, which contains 180 sets. Then, many of the statistical reprocessed databases are used as inputs vector to ANN. Additionally, in this paper 72 different ANN have been established and a comparative analysis between all of them has been accomplished to obtain the effective and optimal techniques. 


\section{Partial Discharge}

The PD is ignited generally if the electrical field strength inside the gaseous inclusion exceeds the intrinsic field strength of the gas. In technical insulation, PD events are the consequence of local field enhancements due to imperfections. Therefore, partial discharges are defined in IEC60270 as:" localized electrical discharges that only partially bridge the insulation between conductors", the PD can or cannot occur adjacent to a conductor. PDs are in general a consequence of local electrical stress concentrations in the insulation or on the surface of the insulation. Generally, such discharges appear as pulses having duration of much less than $1 \mu$. s [11].

\subsection{Partial Discharge Measuring Setup}

In order to generate PD activities in the cable system, conventional PD measurement method was used resonant tuned frequency $50 \mathrm{~Hz}$ AC energizing according to the standard IEC60270 recommendations [11]. The measurements have been taken in the Extra High Voltage Research Centre (EHVRC) - Egypt. The PD experiment laboratory has designed to house a shielded enclosure to prevent external electromagnetic interference which affects the partial discharge measurements. The walls, floor and ceiling consist of modular shielded panels made of particleboards, laminated with zinc coated steel sheets. The shielded hall is equipped with a specially designed system for ventilation and lighting to prevent any electromagnetic interference. As a result, the background noise level is less than $2 \mathrm{pC}$, and the control room consists of similar panels as the shielded enclosure with glass windows for direct view of the test area, test circuit of cable inside a shielded enclosure.

The PD magnitude has been determined at a specified test voltage $1.73 U_{0}$ where $U_{0}$ is the operated voltage. The test was made by raising the test voltage from a value below the specified PD test voltage up to a specified voltage exceeding this voltage. The voltage is then maintained for the specified time, 10 seconds, thereafter, gradually reduced to the mentioned specified PD test voltage. At this voltage level, the voltage is maintained for a specified time and, at the end of this time the specified PD quantity is measured in a given time interval 120 seconds. The constitution of the PD experiment laboratory includes a step up transformer, high voltage reactor, coupling capacitor, a commercial PD detector, and the cable under test as shown in the Figure 1.

In order to save the data measured in the computer memory, all the data were digitally converted. Most available digital PD detectors and have a computerized which can provide two dimensional or 3D-pattern of the PD activity. This paper focuses on the 3D-pattern of measured PD, which obtained the relationship between the PD magnitude ' $q$ ', the pulse count or frequency $\mathrm{H}_{\mathrm{n}}(\varphi, q)$ in the 2 minutes test measuring time, and the power phase angle $(\varphi)$. The PD TE571 detector was applied during the experiments to detect the discharge signals in six XLPE power system cables.

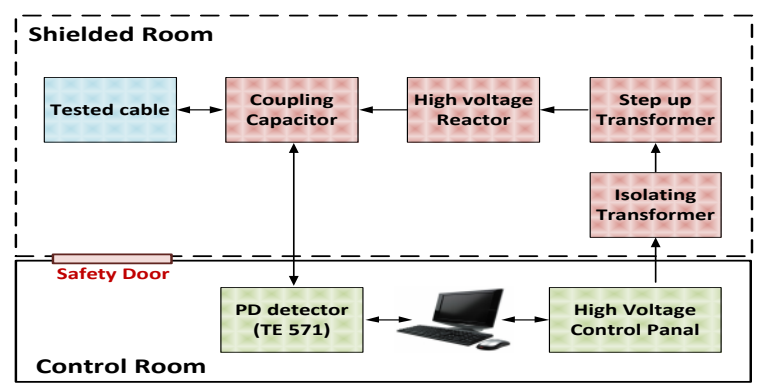

Figure 1: System configuration of the PD detecting system.

\subsection{Cables Sample}

The data were obtained from monitoring 3D-patterns $\left.\mathrm{H}_{\mathrm{n}}(\varphi, q)\right)$ of measured PD in the laboratory. In this paper, the experimental objects are medium power cables that have nominal cross-sectional area $240 \mathrm{~mm}^{2}$, XLPE insulated PVC sheathed XLPE / SCT / PVC / AWA / PVC cable. The cable sample related to three categories of classification; free PD (healthy cable), internal PD (defected cable) and external PD as illustrated in Table1.

Table 1: Cables sample with defect type

\begin{tabular}{|c|c|}
\hline Cable Sample & Defect type \\
\hline $\begin{array}{c}\text { 12/20kV XLPE insulated cable } \\
\text { without defect }\end{array}$ & Free PD \\
\hline $\begin{array}{c}\text { 12/20kV XLPE insulated cable } \\
\text { with cavity artificial defect }\end{array}$ & $\begin{array}{c}\text { Internal PD } \\
\text { only }\end{array}$ \\
\hline $\begin{array}{c}\text { 12/20kV XLPE insulated cable } \\
\text { with cavity and termination } \\
\text { artificial defect }\end{array}$ & $\begin{array}{c}\text { Both Internal } \\
\text { and external } \\
\text { PD }\end{array}$ \\
\hline $\begin{array}{c}\text { 18/30kV XLPE insulated cable } \\
\text { without defect }\end{array}$ & Free PD \\
\hline $\begin{array}{c}\text { Aged 18/30kV XLPE insulated } \\
\text { cable }\end{array}$ & $\begin{array}{c}\text { Internal PD } \\
\text { only }\end{array}$ \\
\hline $\begin{array}{c}\text { 18/30kV XLPE insulated cable } \\
\text { with termination artificial defect }\end{array}$ & $\begin{array}{c}\text { external PD } \\
\text { only }\end{array}$ \\
\hline
\end{tabular}

The cavity artificial defect was established through drilling in the surface of the cable $1 \mathrm{~mm}$ depth below the outer semi-conductive screen by $1 \mathrm{~mm}$ pits sizes to produce internal PD type. The fault in the cable termination as artificial defected was established through letting gap between the insulation and conductors to produce external (corona) PD type. Figure 2 shows the cable sample connected with the test system inside shielded enclosure.

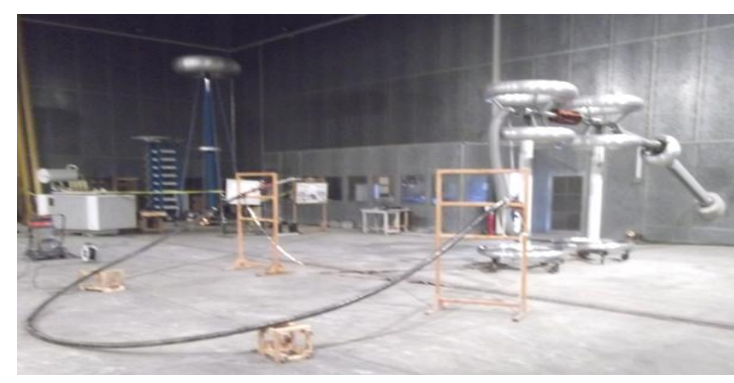

Figure 2: Test circuit inside shielded enclosure 


\section{Cable Testing Results and Data Acquisitions}

This section illustrates the PD measuring result of testing cable sample, and the technique used to create the PD database.

\subsection{Results of Cable Testing}

The PD pulses are grouped by $50 \mathrm{~Hz}$ sine wave phase angle and, consequently, the voltage cycles are divided into phase windows representing the phase angle axis $(0$ to $360^{\circ}$ ). A strong relationship has been found between the features of measuring patterns and the type of defect causing them. The shape of the PD pattern is characteristic for each type of defect.

Therefore, only an expert can use pattern recognition to classify the defect types according to the PD pattern of the visual system. However, success with this visual system depends largely on the long experience of the engineers on the work test with valuable information, often raise visual observation are contrary to the truth of PD sources.

The typical measurement results of $3 d$-pattern $\mathrm{H}_{n}(\varphi, q)$ of testing cables of $12 / 20 \mathrm{kV}$ at three cases: healthy/free PD, defected /internal PD, and defected /corona PD as shown in Figures 3, 4, and 5, respectively. Similarly, the results of testing cables of $18 / 30 \mathrm{kV}$ at three cases healthy/free PD, defected /internal PD, and defected /corona PD as shown in Figures 6, 7, and 8, respectively.

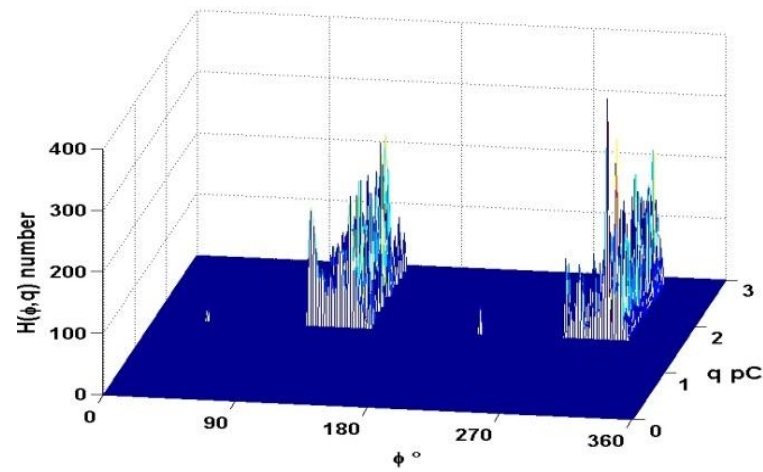

Figure 3: 3d-pattern $H_{n}(\varphi, q)$ processed in $12 / 20 \mathrm{kV}$ XLPE healthy cable sample

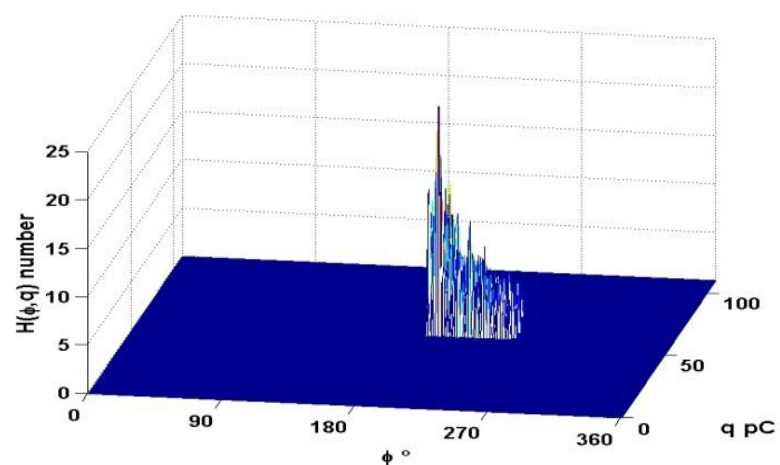

Figure 4:3d-pattern $\mathrm{H}_{\mathrm{n}}(\varphi, q)$ processed for internal partial discharge in $12 / 20 \mathrm{kV}$ XLPE defected cable sample

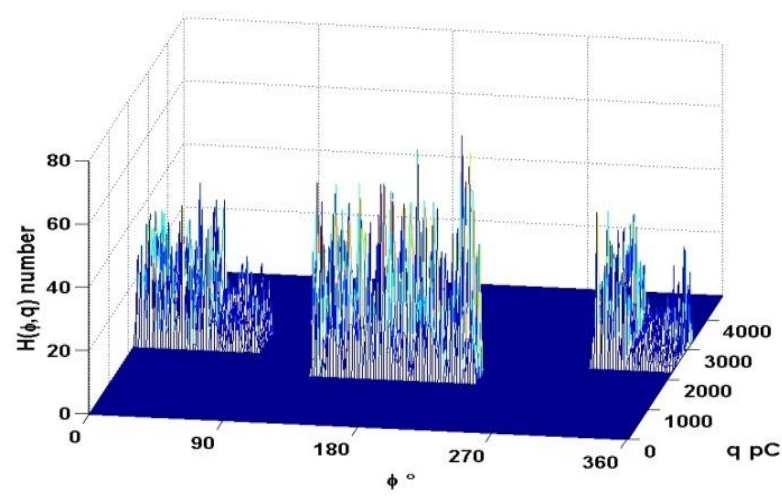

Figure 5: 3d-pattern $H_{n}(\varphi, q)$ processed for corona partial discharge in 12/20 kV XLPE defected cable sample

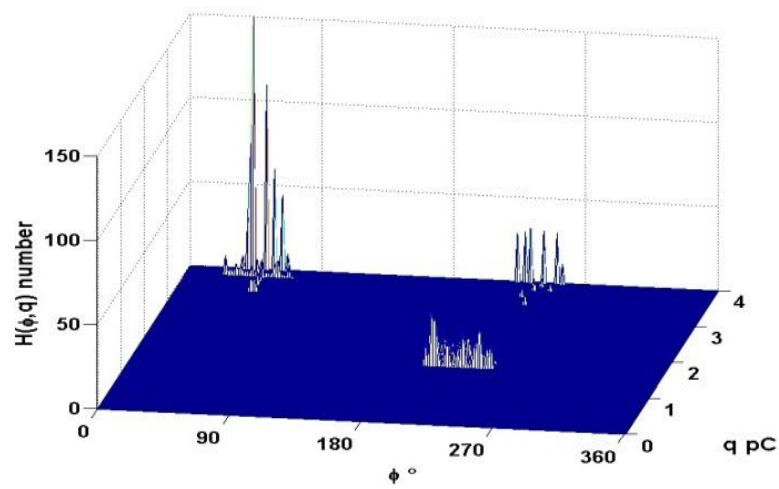

Figure 6: 3d-pattern $H_{n}(\varphi, q)$ processed in $18 / 30 \mathrm{kV}$ XLPE healthy cable sample

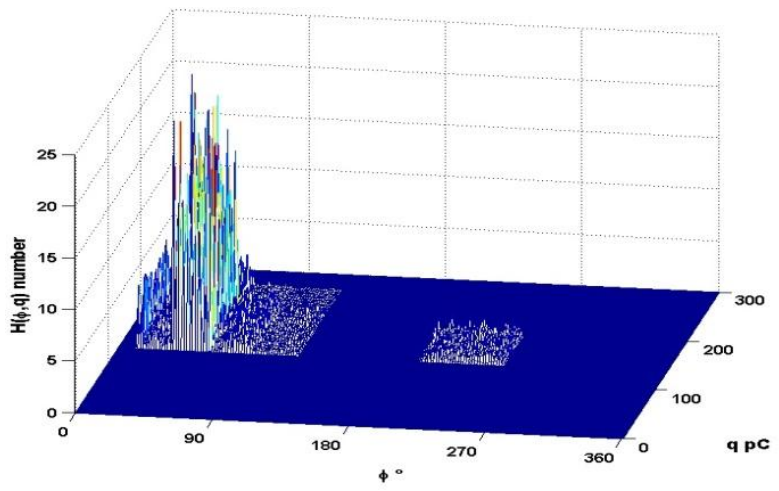

Figure 7:3d-pattern $H_{n}(\varphi, q)$ processed for internal partial discharge in $18 / 30 \mathrm{kV}$ XLPE defected cable sample

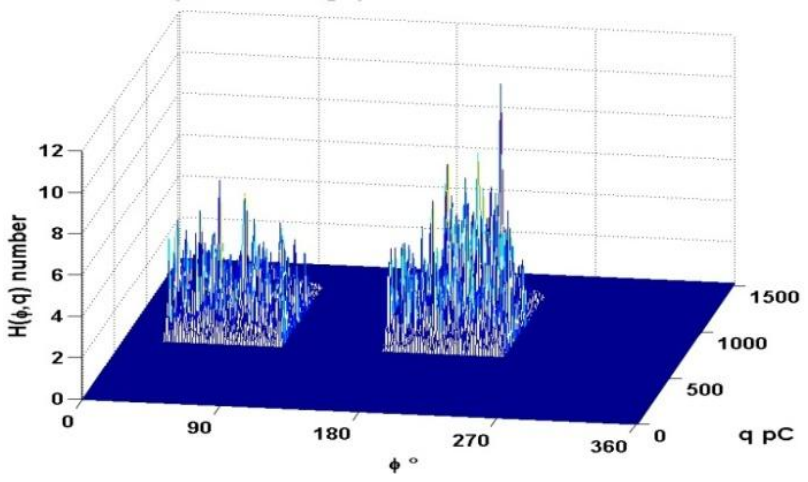

Figure 8: 3d-pattern $H_{n}(\varphi, q)$ processed for corona partial discharge in 18/30 kV XLPE defected cable sample 
The PD characterizes a random phenomena along the phase angle in the situations of testing sample without defect in healthy conditions as shown in Figures 3 and 6. It can be seen that the maximum magnitude of $\mathrm{PD}$ is less than 5pC as recommended in IEC 60270.

Internal discharge occurs in the $1^{\text {st }}$ and $3^{\text {rd }}$ quarter cycle referring to the phase angle of the applied voltage, and normally takes place near the zero crossing ( 0 degree and 180 degree) of the power cycle. Moreover, the PDs activity in the positive cycle is less than the negative cycle as shown in Figure 4 compared to Figure 7. This is because the availability of electrons from the electrode under negative applied voltage, which causes more electron avalanches in the situation of presence artificial cavity in cable sample.

In contrast, the corona discharge starts at the peak of negative cycle, and the discharge magnitude is very asymmetric in positive and negative half cycles as shown in Figures 5 and 8 . When the point electrode is at the high voltage side then the discharges are first ignited at negative voltage. It is clear from Figure 5 that in addition to corona discharge there is some internal PD.

\subsection{PD Data Acquisitions}

In order to obtain ANN classification, this work has created 30 quit similar sets of 3d-patterns for each tested cable by virtue of advanced programs, such as MATLAB program. Therefore, there are 180 sets of $3 d-$ pattern, which has been classified into three categories:

-Healthy cable has free PD

- Defected cable has internal PD

-Defected cable has external PD

The speedy computers and rapid developments in techniques and pattern recognition techniques using ANN automated classification of PD sources are quick and effective.

\section{ANN Based Pattern Recognition and Classification}

The advantages of using ANNs for PD pattern recognition and classification are the ability to estimate the nonlinear relationship between the input data and desired outputs, and faster generalization capability, therefore ANNs may be learnt without difficulty even at various PD patterns. Pattern recognition can be implemented by using a feed-forward scheme as shown in Figure 9.

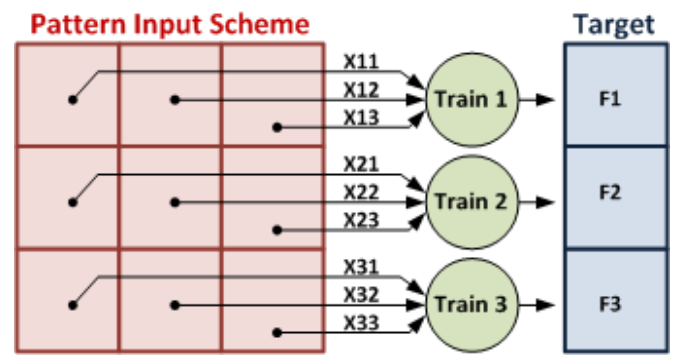

Figure 9: Feed-forward pattern recognition
During training of neural networks, the network is adjusted to associate outputs with input patterns. So, that a particular input leads to a specific target output. Such a situation is shown in Figure 10.

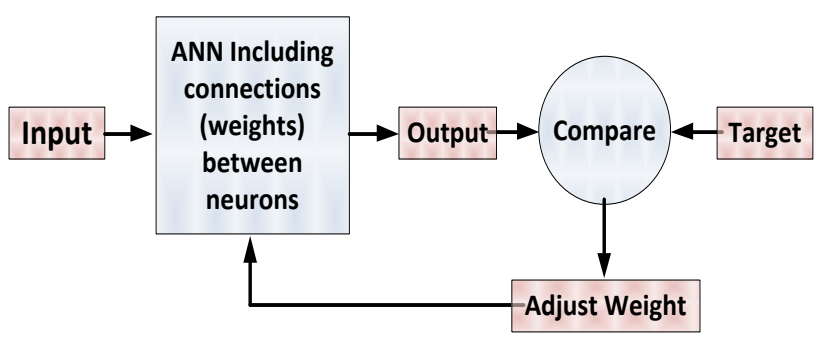

Figure 10: Adjusted weight neural networks

Typically, many such input/target pairs are needed to train a network. In this paper, Artificial Neural Network (ANN) has been built to classify inputs into a set of target categories such as free PD, internal PD and external PD for XLPE power system. Neural Network Pattern Recognition Tool in MATLAB is used in the proposed technique.

\section{Pre-processing and Techniques of Extraction Statistical Feature}

In previous studies, the acquired data of 3d-patterns was directly fed to the input of the ANN for PD recognition in the form of raw $\mathrm{PD}$ pattern. In those approaches, the input layer to ANN was in a large dimension and the main drawbacks are that the structure of the ANN has a great number of neurons with connections, and time-consuming in training. In order to improve the performance, pre-processing schemes that extract relevant statistical features from the raw PD patterns are presented for the proposed ANN-based classifier. There are many statistical methods to evaluate the importance of features such as Fractal geometry [12]. Particularly, in this work, the mean, the standard deviation, the coefficient of variation, skewness, and kurtosis are considered. The block scheme of the proposed approach is shown in Figure 11.

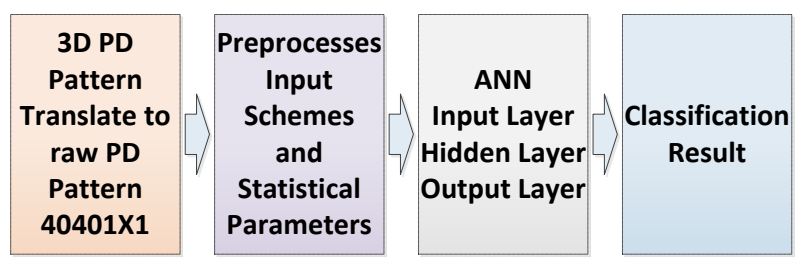

Figure 11: Block scheme of proposed approaches

The output vector of the ANN-based classifier of three categories (Free PD, Internal PD, and Corona PD) will be size as $3 \times 1$ acording to their categories. The input scheme size will be changed according to the extraction technique of statistical feature. For example, as shown in Figure 12, the 8 windows each window phase width is $45^{\circ}$, the statistical features will be extracted for each window to obtain input scheme size $8 X 1$. 


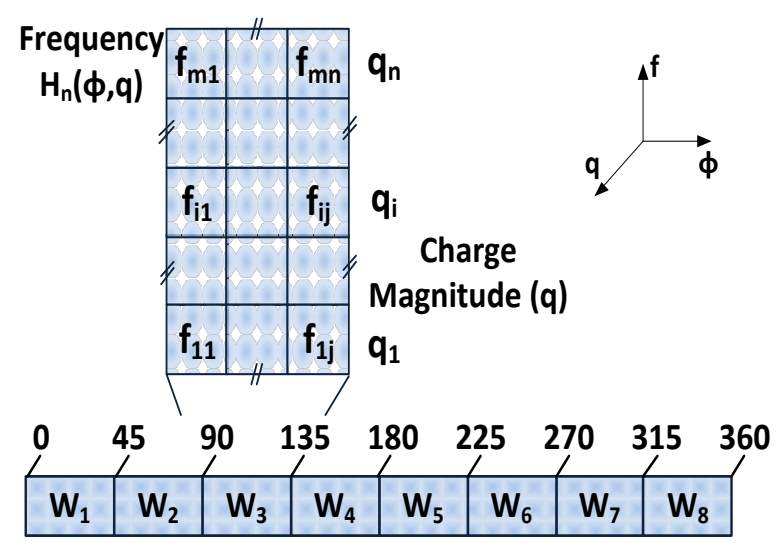

Phase Angle $\phi$

Figure 12: Pre-processing 8-window input scheme of ANN at phase width $\mathbf{4 5}^{\circ}$

The extraction technique is the method used to calculate statistical parameter from 3D- PD pattern. This is achieved by dividing the phase angle axis $\left(0\right.$ to $\left.360^{\circ}\right)$ to $1,2,4,8,10,12,16,18,20,24,30$ or 36 windows, with the different widths of the phase window. There are 12 techniques to extract the statistical features in this work. The values of every phase window calculated by using following standard statistical parameter as in [13]:

1) Mean $(\mu)$ : this gives an estimate of the central value around the distribution cluster as:-

$$
\mu_{q t}=\frac{\sum_{i=1}^{m} \sum_{j=1}^{n} f_{i j} q_{i}}{\sum_{i=1}^{m} \sum_{j=1}^{n} f_{i j}}
$$

2) Standard Deviation $(\sigma)$ : this gives an estimate of the "width" of a distribution around its mean value as :-

$$
\sigma_{q t}=\sqrt{\frac{\sum_{i=1}^{m} \sum_{j=1}^{n} f_{i j}\left(q_{i}-\mu_{q t}\right)^{2}}{\sum_{i=1}^{m} \sum_{j=1}^{n} f_{i j}}}
$$

3) Coefficient of Variation (CV): this is a quantity that measures of the dispersion of distribution points in a data series around the mean as:-

$$
C V=\frac{\sigma}{\mu}
$$

4) Skewness (SK): this is a quantity that measures the degree of asymmetry of a distribution around its mean value as:-

$$
S k_{q t}=\frac{\sum_{i=1}^{m} \sum_{j=1}^{n} f_{i j}\left(q_{i}-\mu_{q t}\right)^{3}}{\sigma_{q t}^{3}\left(\sum_{i=1}^{m} \sum_{j=1}^{n} f_{i j}\right)}
$$

5) Kurtosis $(\mathrm{Ku})$ : this is a quantity that measures the degree of peakedness or flatness of a distribution relative to the normal distribution as :-

$$
K_{q t}=\frac{\sum_{i=1}^{m} \sum_{j=1}^{n} f_{i j}\left(q_{i}-\mu_{q t}\right)^{4}}{\sigma_{q t}^{4}\left(\sum_{i=1}^{m} \sum_{j=1}^{n} f_{i j}\right)}
$$

6) Maximum value of charge as:-

$$
\mathrm{q}_{\mathrm{j} \_\max }=\max \{\mathrm{qi}\} \text { for } \mathrm{i}=1, . ., \mathrm{n} \text { at } \mathrm{j} \text {-column }
$$

$$
\mathrm{f}_{\mathrm{ij} \_\max }=\mathrm{f}_{\mathrm{ij}} \text { at } \mathrm{q}_{\mathrm{j} \_ \text {max }}
$$

\section{RESULTS}

In this section, the results of data pre-processing which involves the statistical features extracted from the 3DPD patterns are presented. Moreover, the performance of ANNs is investigated to obtain the optimal partial discharge pattern classification.

\subsection{Results of the Pre-processing data}

In Figures 13 through 18, the different six statistical properties are shown via the different phase windows. In these figures, the pre-processing input vectors are obtained based on the case of eight windows scheme for different cables, each window covers $45^{\circ}$ of the phase resolved pattern.

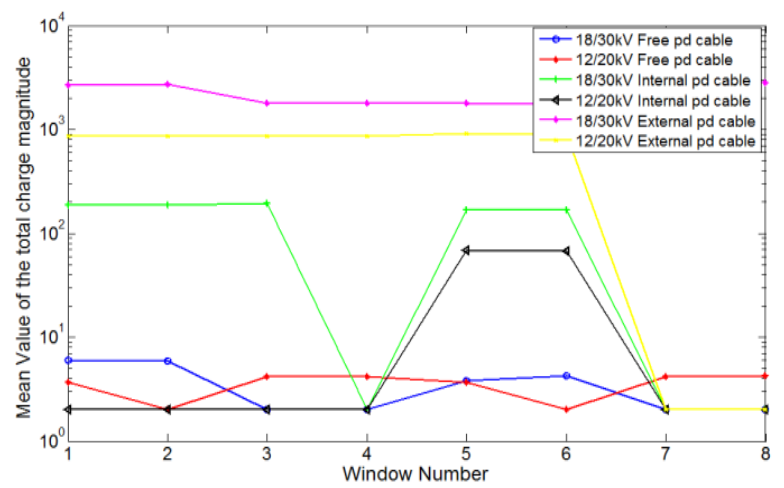

Figure 13: 8-window input scheme of ANN by using mean value

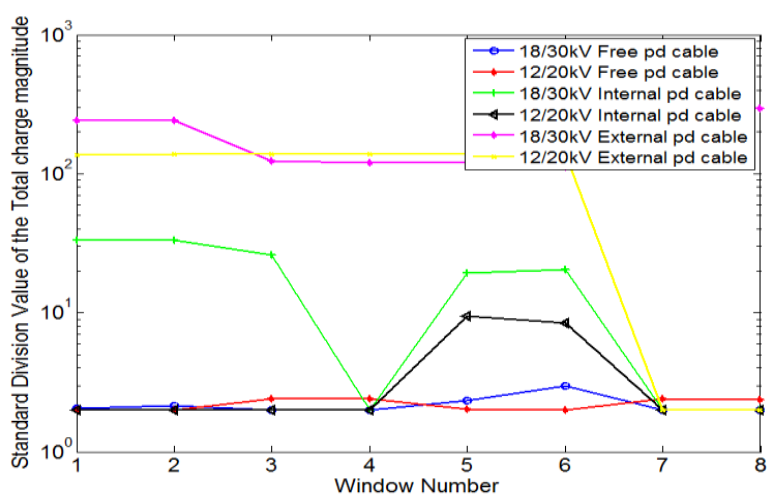

Figure 14: 8-window input scheme of ANN by using standard deviation value. 


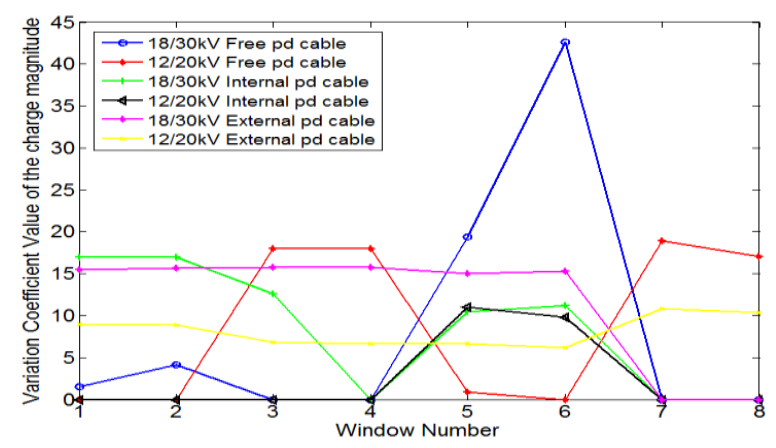

Figure 15: 8-window input scheme of ANN by using variation coefficient value.

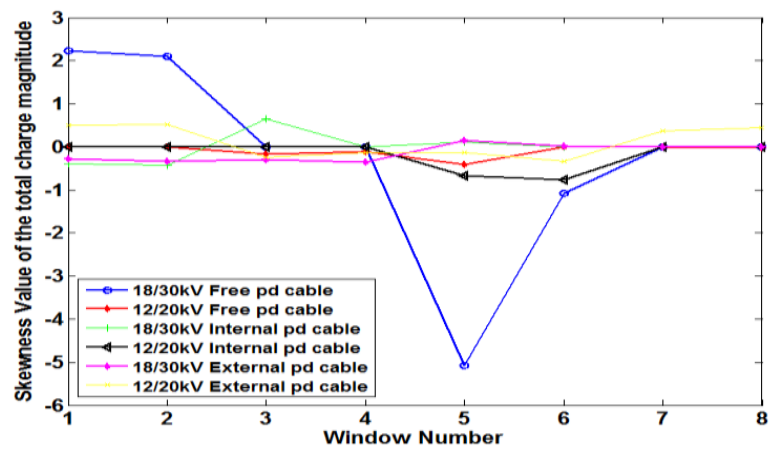

Figure 16: 8-window input scheme of ANN by using skewness value

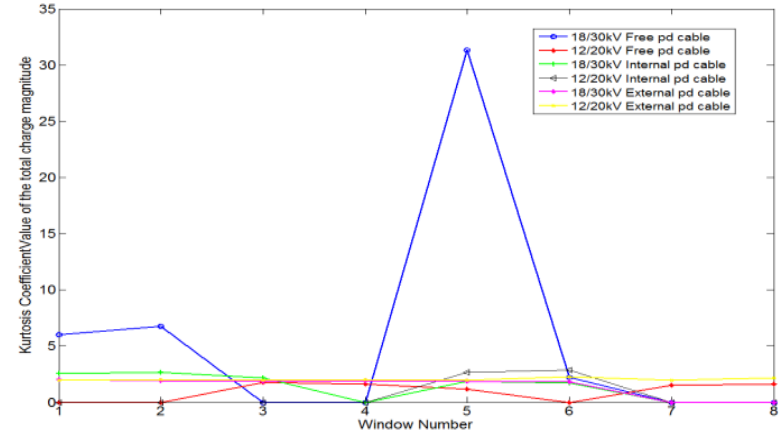

Figure 17: 8-window input scheme of ANN by using Kurtosis value.

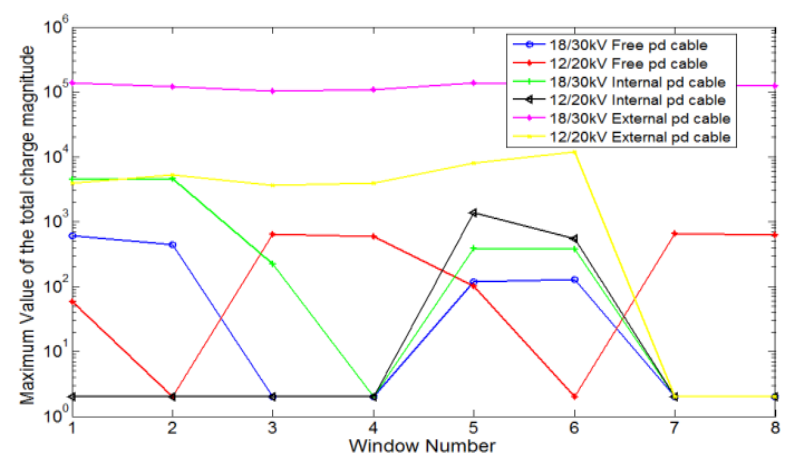

Figure 18: 8-window input scheme of ANN by using Maximum value.

It is depicted from these figures that, the discrimination between the different cables through various statistical properties depends on the variation of these properties. As noted in Figure 19 the increase of phase windows number leads to more pattern discriminations. However, both the number of neurons and the ANN computational time will increase.

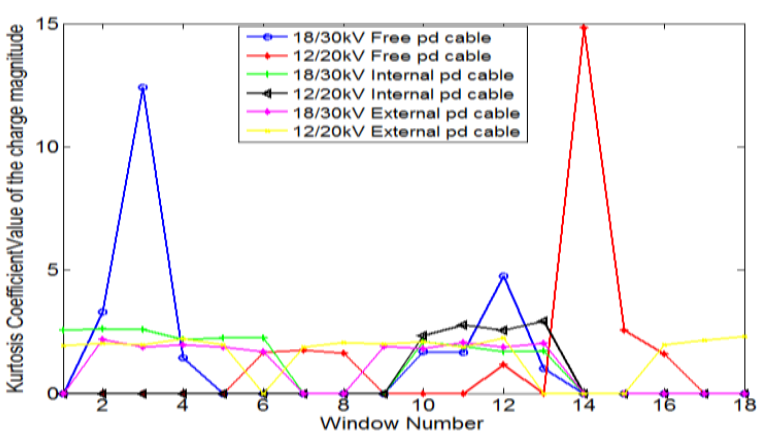

Figure 19: 18-window input scheme of ANN by using kurtosis value.

\subsection{Results of the ANNs performance}

To evaluate the performance of the ANNs, two parameters are used: the mean square error and the accuracy of neural network (the rate of success classifications), which shows various types of errors that occurred in the final trained network. The application is randomly divided the input vectors and target vectors into three sets:

1) $70 \%$ is used for training (126 Samples). The network is adjusted according to its error.

2) $15 \%$ is used to validate (27 Samples). These are used to measure network generalization, and to halt training when generalization stops improving.

3) The last $15 \%$ is used as a completely independent test of network generalization (27 Samples).

Table 2 shows the classified results of the proposed approach. It is clear that the accuracy rates of the proposed approach are quite high about $100 \%$ for training, validation and testing sets. It is obvious that the proposed approach has a strong generalized capability. On the other hand, the classified results of the schemes 1 (single window) have a bad accuracy with all parameters; also, the maximum statistical parameter exhibits the lowest accuracy among all the parameters.

Due to the perfect recovery results in Table 2, MSE metric is used to evaluate the performance of different statistical properties as in Figure 20. MSE indicate the various types of errors that occurred in the final network, and the accuracy of neural network, which has been calculated as the rate of success classifications.

Table 2: Accuracy percentage of classification results

\begin{tabular}{|c|c|c|c|c|c|c|}
\hline $\begin{array}{c}\text { No. of } \\
\text { windows }\end{array}$ & $\boldsymbol{\mu}_{q t}$ & $\sigma_{q t}$ & $\mathbf{C V}$ & $\mathbf{S K}_{\mathbf{q t}}$ & $\mathbf{K}_{\mathbf{q t}}$ & $\mathbf{q}_{\mathbf{m a x}}$ \\
\hline $\mathbf{1}$ & 83 & 67 & 94 & 71 & 89 & 82 \\
\hline $\mathbf{2}$ & 100 & 100 & 99 & 80 & 83 & 82 \\
\hline $\mathbf{4}$ & 100 & 100 & 100 & 100 & 100 & 88 \\
\hline $\mathbf{8}$ & 100 & 100 & 100 & 100 & 100 & 80 \\
\hline $\mathbf{1 0}$ & 100 & 100 & 100 & 100 & 100 & 81 \\
\hline $\mathbf{1 2}$ & 100 & 100 & 100 & 100 & 99 & 74 \\
\hline $\mathbf{1 6}$ & 100 & 100 & 100 & 100 & 100 & 95 \\
\hline $\mathbf{1 8}$ & 100 & 100 & 100 & 100 & 100 & 97 \\
\hline $\mathbf{2 0}$ & 100 & 100 & 100 & 100 & 100 & 92 \\
\hline $\mathbf{2 4}$ & 100 & 100 & 100 & 100 & 100 & 90 \\
\hline $\mathbf{3 0}$ & 100 & 100 & 100 & 100 & 100 & 92 \\
\hline $\mathbf{3 6}$ & 100 & 100 & 100 & 100 & 100 & 93 \\
\hline
\end{tabular}




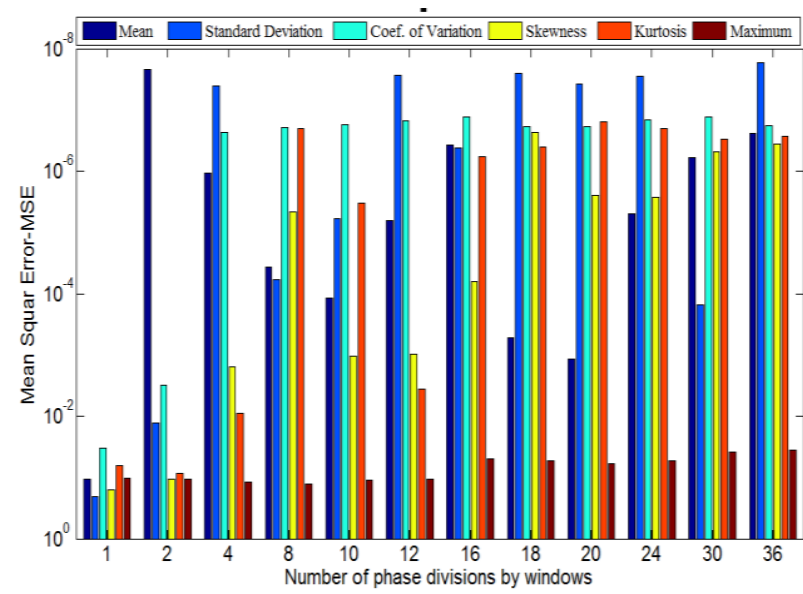

Figure 20: MSE for all established ANN's with statistical parameters and different schemes.

Figure 20, also, illustrates that the standard deviation and the mean parameters are sufficient for perfect classification with low MSE lower than 0.01 even at low number of windows schemes $(2,4)$.

In addition, all statistical parameters except the maximum achieve low MSE in ranges of $10^{-4}$ using 8 or higher windows schemes. These observations of Figure 20 are assured the obtained results in Table 2.

\section{Conclusion}

This paper proposed an approach based on pattern classification of different PD defect such as internal and external (corona) PD commonly occurring in XLPE medium voltage cable. This proposed approach presented a comparison between different statistical techniques and schemes extracted from the measured 3D-PD patterns.

The use of extracting feature statistics from 3D-PD patterns can reduce the size of the PD pattern database and save time processing of classification. The ANNbased PD pattern classification is very effective in recognizing the defects of XLPE cable through multiple phase windows. As, the method has a high accuracy rate of $100 \%$ and $10^{-4}$ MSE using mean, standard deviation and coefficient of variation at scheme 4 windows is implemented on is very useful.

\section{Reference}

[1] Jiang Lei, Zhu Deheng, Li Fuqi, Tan Kexiong, Qin Gangli, Jian Xianhe, Wang Changchang, T.C Cheng, Partial Discharge Pattern Recognition of Insulation Models of Power Transformer, Proceedings of The 6th International Conference on Properties and Applications of Dielectric Materials, June 21-26, (2000), pp.129-132.
[2] S. Boonpoke and B. Marungsri" Pattern Recognition of Partial Discharge by Using Simplified Fuzzy ARTMAP", World Academy of Science, Engineering and Technolog China, pp 212-219, September 2010.

[3] B. Karthikeyan, S. Gopal and S. Venkatesh, A heuristic complex probabilistic neural network system for partial discharge pattern classification, J. Indian Inst. Sci., Sep.-Oct. 2005, 85, 279-294.

[4] Evagorou, D., Kyprianou, A., Lewin, P.L., Stavrou, A., Efthymiou, V., Georghiou, G.E.: 'Classification of partial discharge signals using probabilistic neural network', IEEE Int. Conf. on Solid Dielectrics (ICSD), July 2007, p. 609-615

[5] M. M. A. Salama, and R. Bartnikas, "Determination of Neural Network Topology for Partial Discharge Pulse Pattern Recognition," IEEE Transactions on Neural Networks, Vol. 13, pp. 446-456, 2002.

[6] Po-Hung Chen, Hung-Cheng Chen, An Liu, LiMing Chen, Pattern recognition for partial discharge diagnosis of power transformer, International Conference on Machine Learning and Cybernetics (ICMLC), 2010 (Volume:6 ), 1114 July 2010,p. 2996 - 3001.

[7] Sahoo, N., Salama, M., Bartnikas, R.: 'Trends in partial discharge pattern classification: a survey', IEEE Trans. Dielectr. Electr. Insul., 2005, 12, (2), p. $248-264$

[8] Hung-Cheng Chen, PO-Hung Chen, and MengHui Wang " Partial Discharge Classification Using Neural Networks and Statistical Parameters", International Conference on Instrumentation, Measurement, Circuits \& Systems, April 15-17, 2007,p.p 84-88.

[9] Mang-Hui Wang, Partial Discharge Pattern Recognition of Current Transformers Using an ENN, IEEE Transaction on Power Delivery, VOL. 20, NO. 3, JULY 2005.

[10] Hung-Cheng Chen, Po-Hung Chen, Chien-Ming Chou, "3-D Partial Discharge Patterns Recognition of Power Transformers Using Neural Networks", " Advances in Neural Networks ISNN 2006, pp. 1324-1331.

[11] High-Voltage Test Techniques Partial Discharge Measurements, IEC 60 270, 2001.

[12] Elsayed M. Elrefaie, M. K. Abd Elrahman, Sherif Ghoneim, Ahmed Bakr, Classification of Partial Discharge Patterns Using Fractal Geometry, International Journal of Engineering Research and Applications (IJERA) ISSN: 2248-9622,Vol. 2, Issue 5, pp.1010-1015, October 2012.

[13] K.X. Lai; B.T. Phung; T.R. Blackburn; N.A. Muhamad, Comparison of partial discharge activity in mineral oil and bio-degradable oil, 2007 Australasian Universities Power Engineering Conference, AUPEC.\& thinsp, 2007. 